Technical notes

\title{
A three dimensional drive system for use with fillable emission phantoms for SPECT and PET imaging
}

\author{
R. Darwesh ${ }^{\text {a,b,* }}$, D. Clay ${ }^{\text {c }}$, P.D. Hay ${ }^{\text {c }}$, C. Kalirai ${ }^{c}$, H. Rassoulian ${ }^{c}$, A. Pitiot ${ }^{\text {d }}$, A.C. Perkins ${ }^{\text {a,c }}$ \\ ${ }^{a}$ Radiological and Imaging Science, School of Clinical Science, University of Nottingham, Nottingham, UK \\ ${ }^{\mathrm{b}}$ Department of Medical Physics, King Abdul-Aziz University, Jeddah, Saudi Arabia \\ ${ }^{\mathrm{c}}$ Department of Medical Physics and Clinical Engineering, Nottingham University Hospitals NHS Trust, Nottingham, UK \\ ${ }^{\mathrm{d}}$ School of Psychology, University of Nottingham, UK
}

\section{A R T I C L E I N F O}

\section{Article history:}

Received 18 January 2012

Received in revised form

13 June 2012

Accepted 16 June 2012

Available online 12 July 2012

\section{Keywords:}

Phantom drive system

4D imaging

Thoracic imaging

Motion artefacts

\begin{abstract}
A B S T R A C T
Respiratory motion artefacts pose significant challenges for imaging of the lung and thorax. Dynamic phantoms have previously been applied to the study of respiratory motion, however, most moving platforms have been capable of movement in either one or two dimensions only. We describe a moving platform suitable for SPECT-CT and PET-CT imaging. The platform allows a fillable emission phantom to simulate rigid motion in three dimensions. Elliptical periodical motion of $1.5 \mathrm{~cm}$ in all three orthogonal planes was simulated using a series of cams moving a baseplate up and across a slope of $45^{\circ}$. The frequency of movement can be varied manually between 5 and 25 cycles per minute in a known calibrated and reproducible manner (This encompasses the range of physiological respiratory motion). Preliminary studies demonstrated that the phantom can be used to identify motion parameters and for the qualitative assessment of motion blurring in reconstructed images.
\end{abstract}

(c) 2012 Associazione Italiana di Fisica Medica. Published by Elsevier Ltd. All rights reserved.

\section{Introduction}

It is well known that respiration-induced anatomic motion is an important source of geometric or positional uncertainty when imaging hepatic and thoracic disease sites [1-3]. Respiratory motion artefacts pose significant challenges for modalities such as PET and SPECT [1]. The presence of respiratory motion during PET or SPECT scanning causes blurring of the moving objects in the images [4]. This blurring has a number of confounding effects including degradation of spatial resolution [4,5], reduction in overall contrast, loss of quantitative accuracy when assessing tumour uptake [4] and reduction in lesion detectability. Respiratory motion could impair the detection of pulmonary embolism particularly at the lung bases in planar and SPECT ventilation/perfusion images [6]. In addition it can introduce difficulties when co-registering SPECT or PET with CT data which is typically acquired using a breath-hold technique [7].

The effect of the motion problem in diagnostic imaging and radiotherapy has been studied extensively in recent years utilising physical phantom data, computer simulation phantom data and patient data. A number of solutions have been suggested with different assumptions made using each approach $[2,8]$. However,

\footnotetext{
* Corresponding author. Radiological and Imaging Science, School of Clinical Science, University of Nottingham, Nottingham, UK.

E-mail address: mgxrd@nottingham.ac.uk (R. Darwesh).
}

there is still a need to gain further practical knowledge of the imaging problems. A moving phantom should be of great value in determining the optimal image acquisition parameters and data processing techniques to overcome the problems caused by respiratory motion since the motion parameters are implicit in the construction and are therefore accurately known.

This technical note describes the design and initial use of a 3D phantom drive system that provides motion in three dimensions. The system was designed for gamma camera and PET imaging but would also be suitable for use with CT scanners.

\section{Material and methods}

\section{Description of the $3 D$ phantom drive system}

The 3D phantom drive system was designed, constructed, calibrated and safety tested in the Engineering Design and Research Support Unit, in Clinical Engineering at the Queen's Medical Centre Campus of Nottingham University Hospitals, Nottingham.

The 3D phantom drive system was designed to enable an anthropomorphic phantom to move in a periodical elliptical trajectory with set movement of $1.5 \mathrm{~cm}$ in three orthogonal axes (Fig. 1). This amplitude of movement was selected following a literature review of previously published papers which indicated this to be representative of average lung movement. A variable 


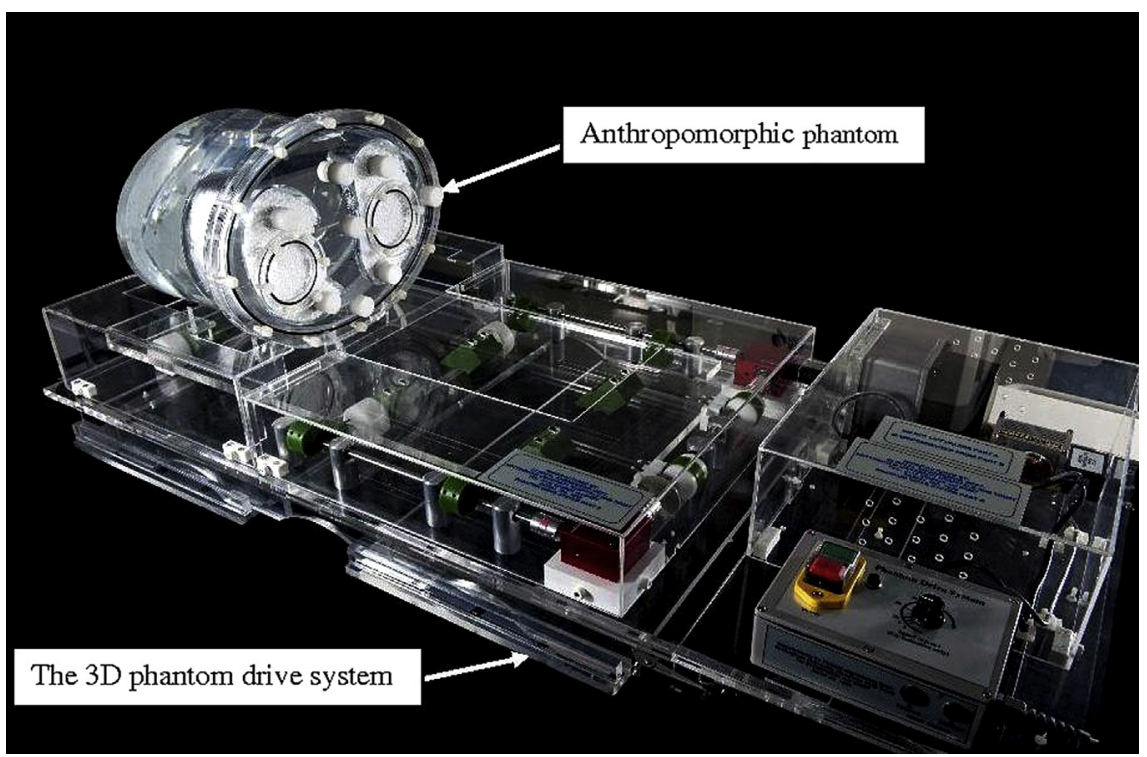

Figure 1. The 3D phantom drive system with an anthropomorphic phantom in place.

control altered the frequency between 5 and 25 cycles per minute (the typical frequency observed during respiration is between 10 and 20 cycles per minute [9]). This frequency is achieved by means of an electronic speed control system attached to the drive motor. The frequency of motion could be varied manually in a known calibrated and reproducible manner during operation.

The 3D phantom drive system consisted of two parts; these were the motor drive unit and the phantom platform (Fig. 2). When in use, the two parts were connected together using mechanical catches. These could be easily detached such that the system can be split into the two component parts for ease of handling and storage. The whole baseplate of the 3D phantom drive system was designed to fit most common types of camera couch. The overall dimension of the phantom drive system was $1160 \mathrm{~mm}$ long, $500 \mathrm{~mm}$ wide, and the system weighs $12 \mathrm{~kg}$. The platform was designed to accommodate phantoms with a nominal weight of up to $12 \mathrm{~kg}$.

The motor drive unit consisted of the motor, power supply, speed control and drive electronics. These were mounted on a baseplate attached to the platform by means of two catches. A mechanical driveshaft connector engaged the motor with the cams.

The motor and drive electronics operated from $24 \mathrm{~V}$ dc supply derived from a power supply mounted on the motor drive unit. The motor control electronics were sited within a metal box together with the motor/gearbox to shield against electromagnetic interference. The motor/gearbox arrangement was capable of turning the camshafts between speeds between 5 and 25 revolutions per minute (rpm).

The $24 \mathrm{~V} \mathrm{dc}$ motor drive system could supply a torque of $4.6 \mathrm{Nm}$ maximum and was designed to detect and compensate for any increase in speed during a cycle.

The second part was the unit, on which the phantom rests, consisted of an acrylic sheet with machined supports for secure placement of the phantom. The platform was attached by means of $4 \mathrm{~mm}$ screws to six triangular ramps, which were positioned beneath the platform equally spaced about its centreline. These in
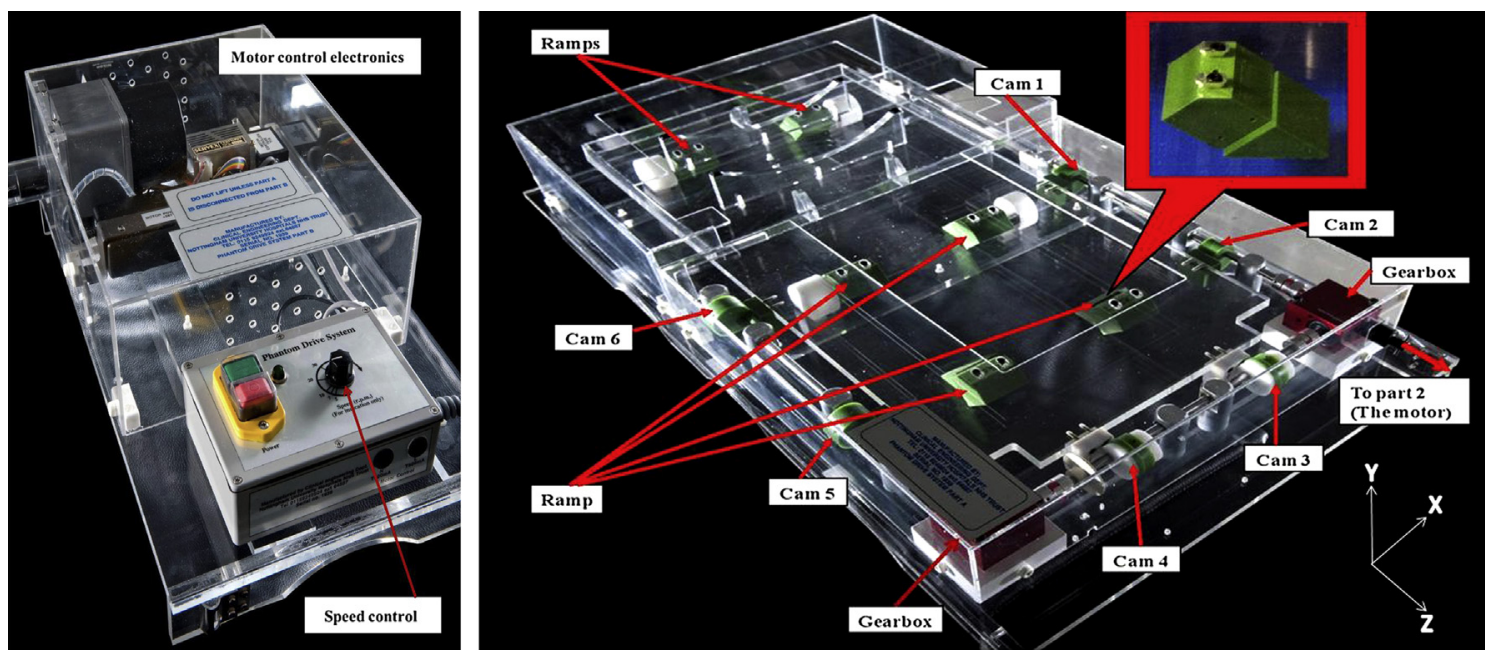

Figure 2. The 3D phantom drive system; Left: The motor drive and switching unit and Right, the 3D platform. 


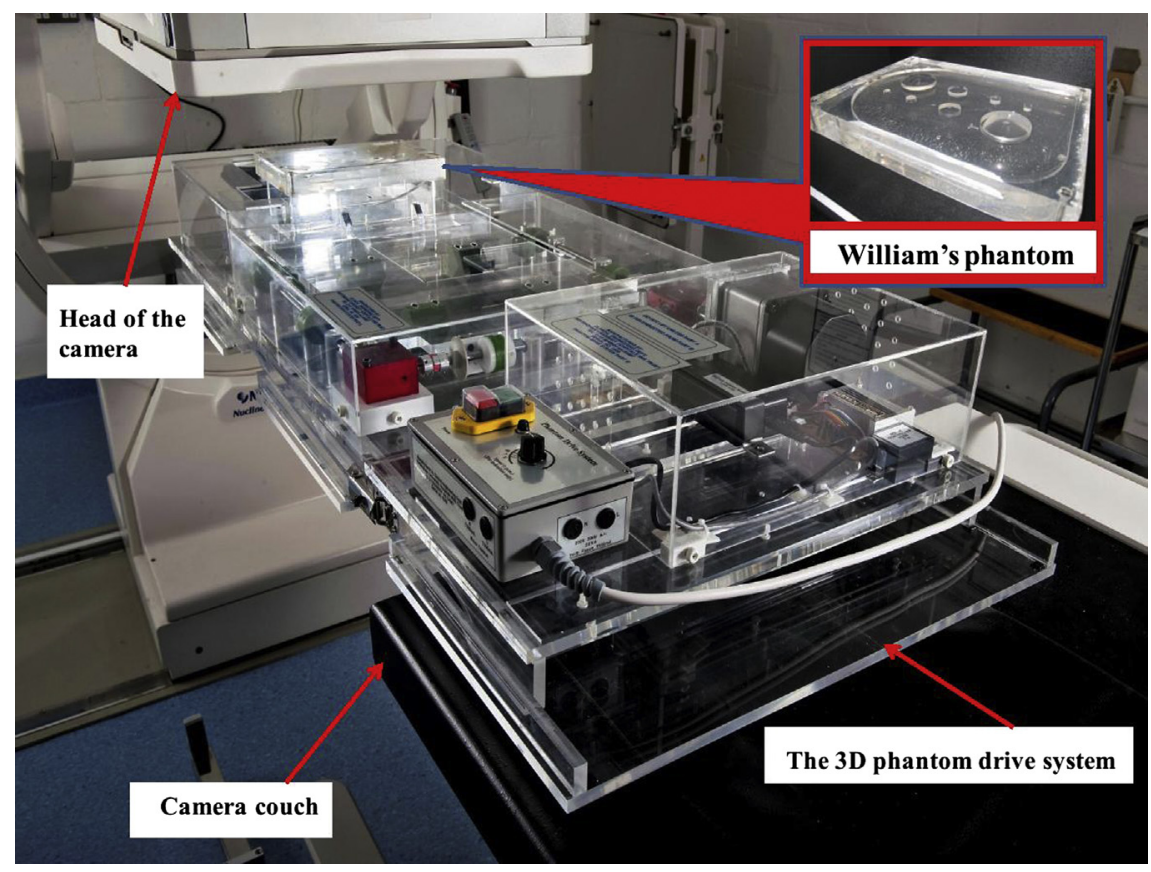

Figure 3. The 3D phantom drive system with the Williams' phantom on the couch of the gamma camera.

turn rested on an equal number of triangular ramps that were attached to the baseplate, also by means of $4 \mathrm{~mm}$ screws.

Movement of the platform on the triangular ramps was facilitated by means of six rotating cams, positioned such that there are two cams on three sides of the platform. Each set of two cams was connected via a right angled gearbox to a $24 \mathrm{~V}$ dc motor.

The cams were set such that one revolution of the motor moves the platform in an elliptical path in the three planes $x, y$, and $z$, by pushing the platform up the ramps, sideways along them, and guiding it as it moves down the ramp.

The motor turned all of the cams at the same time with the same frequency. Cams 1, 2, 5 and 6 push the platform back and forth along horizontal $x$ direction (Fig. 2). At the same time the motion of cams 3 and 4 moved the platform horizontally along the $z$ direction and up the ramps at an angle of $45^{\circ}$, thus moving up in the $y$ plane.

Once at the top of the ramps the platform moves back down the ramps under the control of the motor and via the cams ( 3 and 4). A motor controller compensated for any increase in speed due to gravity. This compensation ensured a smooth drive motion throughout the cycle.

All the cams were mounted on their respective shafts in such a way that the phantom achieved a motion in each of the three planes throughout a sinusoidal motion. The area on which the phantom sits did not contain any metal parts thus avoiding any attenuation and emission scanning artefacts.

\section{Data acquisition}

Two types of data acquisition were used for the preliminary evaluation of the performance of the 3D dynamic phantom drive system these being obtained by SPECT and planar acquisitions.

All planar imaging was carried out in the Academic Research Unit at Queen's Medical Centre Campus of Nottingham University Hospitals, Nottingham, using a single head gamma camera (Mediso, Medical imaging systems, Nucline ${ }^{\mathrm{TM}} \mathrm{X}$-Ring) interfaced to a Hermes nuclear medicine computer (Hermes Medical, London UK). A standard William's phantom consisting of 4 hot circular regions and
4 cold circular regions was used for planar imaging (Fig. 3). Such phantoms have been widely used in the UK but there are few references as to the origin of this test object [10-12]. The phantom cavity was filled with $400 \mathrm{MBq}$ of ${ }^{99 m} T_{\mathrm{C}}$ solution and was placed on the platform of the 3D drive system (Fig. 3) which was placed on the patient couch. A planar static scan was acquired for 5 min without any motion for use as a control in the experiment. Then a further scan was acquired after introducing the motion. The matrix size was selected as $128 \times 128$ to mimic the clinical situation (pixel size of $4.13 \mathrm{~mm}$ ).

SPECT images were acquired in the Nuclear Medicine Department at the Queen's Medical Centre Campus of Nottingham University Hospitals, Nottingham, using a SPECT scanner (Brightview XCT, Philips). A Jaszczak phantom; Benchmark Standard ECT Jaszczak Phantom ${ }^{\mathrm{TM}}$; (Data Spectrum Corp. Surrey, UK) was used (Fig. 4). The Jaszczak phantom is a cylindrical phantom that

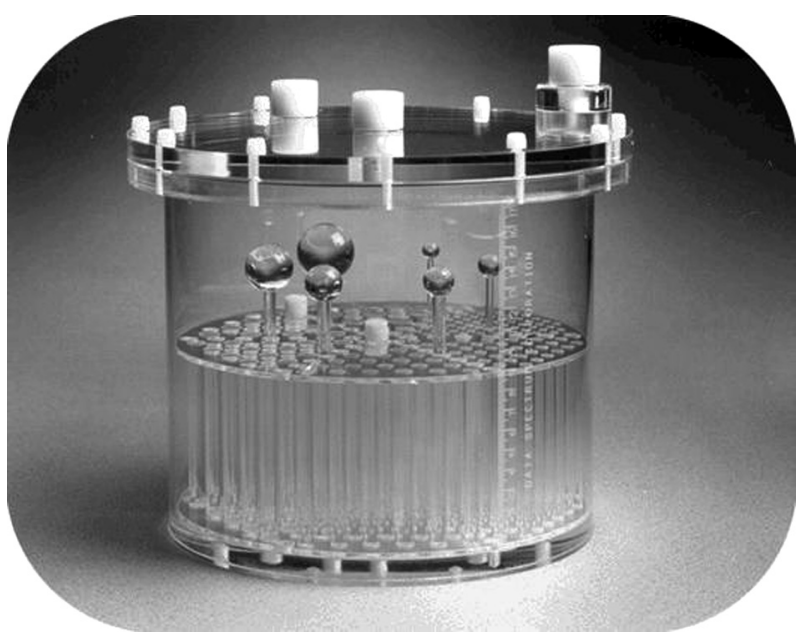

Figure 4. Benchmark Standard ECT Jaszczak Phantom ${ }^{\mathrm{TM}}$. 

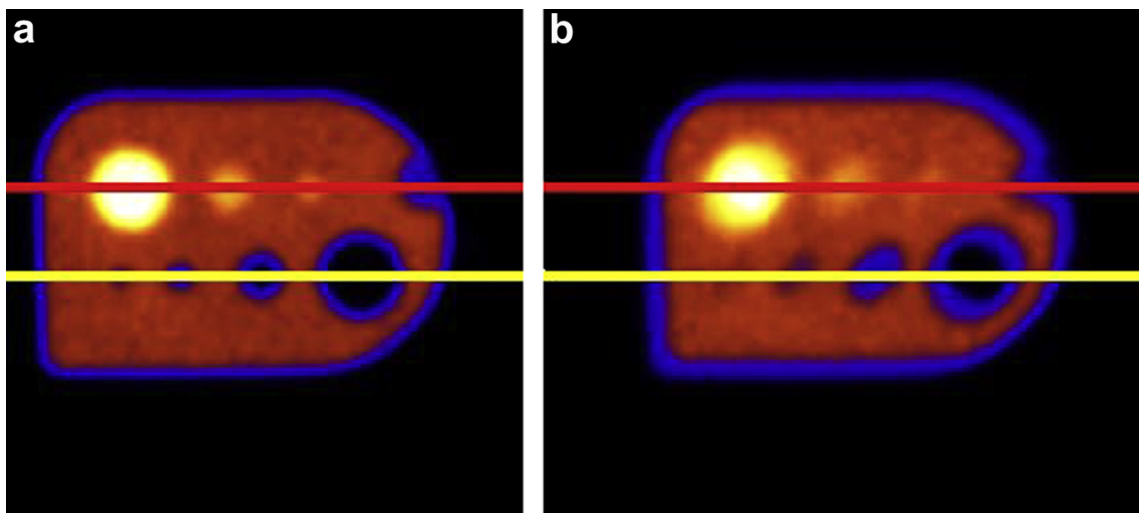

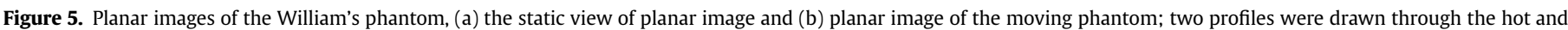
the cold circular regions at the same position in each image.

contains hollow spheres. Five hollow spheres were used with diameters of 15.9, 19.1, 25.4, 31.8 and $38 \mathrm{~mm}$. Each of the spheres were filled with ${ }^{99 m} T_{\mathrm{c}}$ to achieve sphere to background activity ratio of 5:1. The images were acquired using a clinical SPECT-CT protocol. This comprised 60 projections at $25 \mathrm{~s}$ per projection, using a low energy high resolution collimator with auto-contouring of the camera heads. Auto-contouring was used to minimize the collimator-to-phantom distance during rotation of the camera heads. This is the standard method used clinically to reduce attenuation of collected counts during data acquisition.

The Jaszczak phantom was positioned on the platform with the sphere plane perpendicular to the scanning couch. A static SPECT view with the platform stationary was acquired for use as a control. A further SPECT acquisition was acquired with a platform motion using a frequency of 10 cycles per min. The images were reconstructed using an iterative method with attenuation correction (OSEM algorithm with 10 iterations and 4 subsets using a $128 \times 128$ reconstruction matrix size with pixel size of $4.42 \mathrm{~mm}$ ). Following reconstruction a Butterworth filter (cut-off 1.2 cycles $/ \mathrm{cm}$ ) was applied.

\section{Data analysis}

In both the planar and SPECT image data, qualitative comparisons between the static image for the stationary and the moving phantom were performed. In the planar, two line profiles were drawn through the hot circular regions and the cold circular regions in both recorded images (Fig. 5). The profiles from the static image were used as the control. In the reconstructed SPECT data three horizontal profiles were drawn through the five hot spheres in both the static and moving images. The first profile was drawn through the smallest sphere $(15.9 \mathrm{~mm})$, the second profile was drawn through the two middle spheres (38 \& $19.1 \mathrm{~mm}$ ), and the third was drawn through the two bottom spheres (31.8 \& $25.4 \mathrm{~mm}$ ) (Fig. 7).

The effect of the motion was evaluated qualitatively on the basis of the reduction of image blur and reduction in profile width.

\section{Results}

In the preliminary experiments, the capability of the 3D phantom drive system to move accurately in 3 dimensions with $1.5 \mathrm{~cm}$ amplitude throughout the different speed range $(5-25 \mathrm{rpm})$ was determined. This would enable selection of varying speeds for example to investigate different respiration rates.

\section{Planar gamma camera performance test}

The images acquired with motion of the 3D phantom drive system showed distortion and blurring compared to the images acquired with the phantom stationary (Fig. 5(a) and (b)). In the image with motion the third smallest hot circular region and the fourth smallest cold circular region were hardly distinguishable
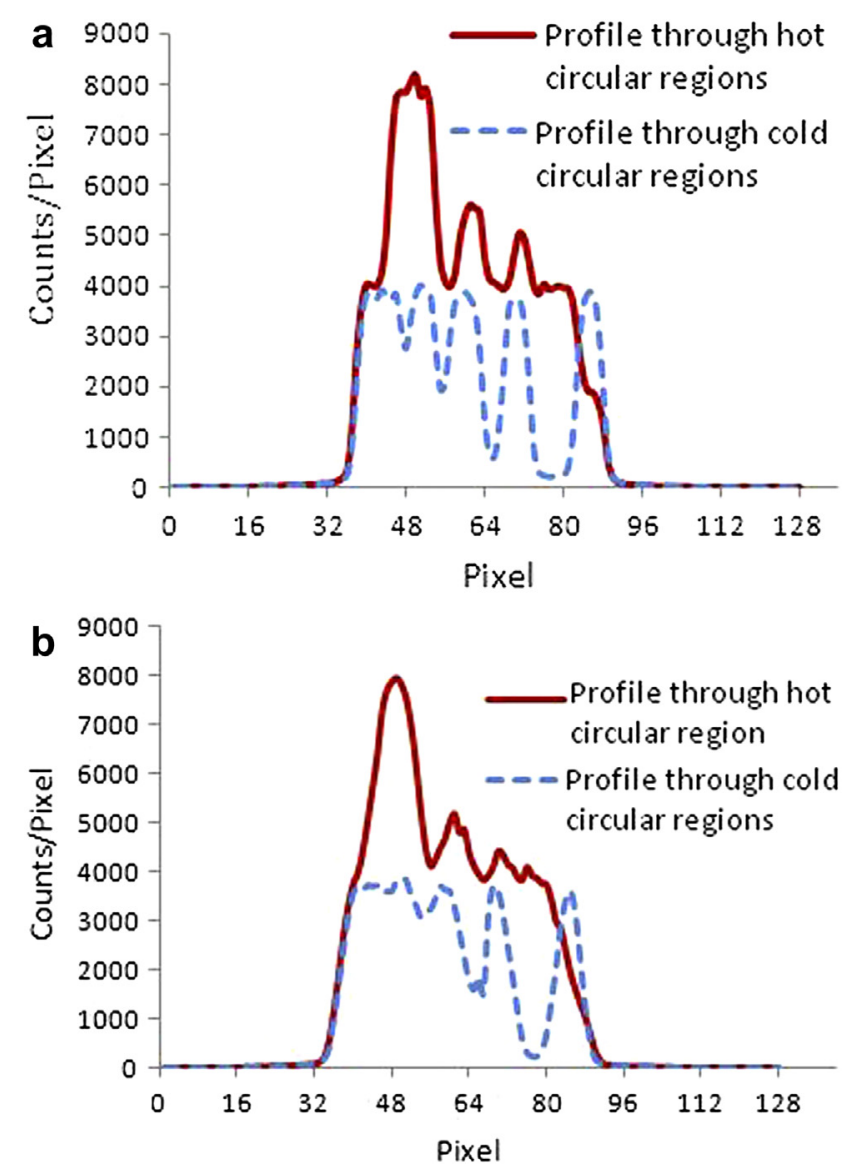

Figure 6. (a) Profiles obtained from the planar image with no motion; and (b) Profiles obtained from the planar image with motion; Red profiles were drawn through the hot circular regions; dashed blue profiles were drawn through the cold circular regions. (For interpretation of the references to colour in this figure legend, the reader is referred to the web version of this article.) 

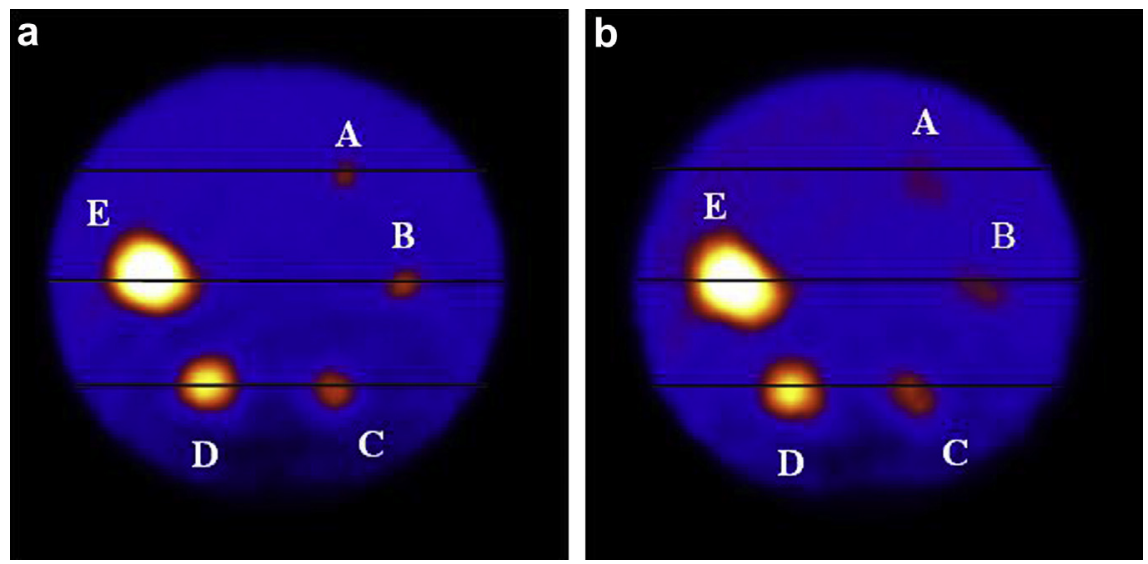

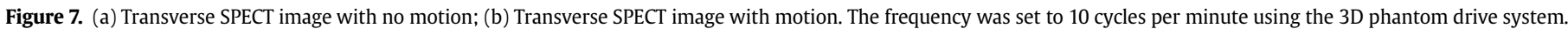

from the background. The activity profiles obtained from this image are shown in Fig. 6(a) and (b).

\section{SPECT performance test}

The effect of the motion on the reduction of image quality is demonstrated in Fig. 7. The image with movement of the phantom showed blurring compared to the static view.

By comparing the profiles of the SPECT images acquired with and without the motion, it is clearly demonstrated how motion reduces the definition of the hot and cold areas (Fig. 8).

\section{Discussion}

To investigate the effects of subject motion it is possible to carry out phantom studies using either test objects or computer simulation software phantoms [2]. Whilst software phantoms can be of great value these do not allow any form of testing of the system acquisition parameters. This communication describes a platform for use with the standard fillable emission phantoms used on PET and SPECT cameras.

A study by Liu et al. investigated the impact of respiratory motion on lung and liver tumours quantification and delineation in PET-CT imaging using physical phantom data, computer simulation phantom data and patients' data [2]. The results show that respiratory motion can have a significant impact on measures derived from PET-CT imaging, depending on motion amplitude, lesion location and size, choice of attenuation map and respiratory pattern, and can cause, on average, $28 \%$ underestimation of lesion standard uptake value, $\mathrm{SUV}_{\max }$, and $130 \%$ overestimation of lesion volume with a threshold at $50 \% \mathrm{SUV}_{\max }$ for a $1 \mathrm{~cm}$ lesion with $11 \mathrm{~mm}$ diaphragm motion. The largest mean $\mathrm{SUV}_{\max }$ error was found to be $\sim 50 \%$ for liver lesions with $16 \mathrm{~mm}$ diaphragm motion. The overestimation of tumour volume as a result of respiratory motion might impact the staging process of the tumour and degrades the anatomic position reproducibility during imaging and necessitates larger margins during radiotherapy planning [1]. The displacement caused by respiratory motions during treatment results in an increase in the treated volume, causes smearing of the dose distribution that defeats the purpose of high precision radiotherapy and leads to a mismatch between the delivered dose distribution and that which was planned [13].

A number of dynamic phantoms have previously been developed to study the effect of respiratory motion on lung or cardiac imaging [13]. However, from our review of the literature to date, we have only found one phantom that appears to be capable of moving in all three dimensions and this has only been used for moving spherical solid phantoms in 4 dimensional CT imaging [14].

In addition to the systems described in Table 1, there are commercially available moving phantoms or platforms, such as the Quasar programmable respiratory motion devices. These are capable of moving a hot spot in only 1 or 2 dimensions and are relatively expensive. In comparison our phantom was made at a cost price of less than EUR1200.

It is important to note that in our current design, the frequency of motion is configurable but set for the duration of the acquisition. This could be regarded as a disadvantage when compared to the Quasar systems that have programmable motion. However in practice, with respiratory gated imaging, irregular motion is commonly rejected during acquisition (similar to "bad beat" rejection in cardiology).

An advantage of our system over the Quasar respiratory motion phantom is that our platform can accommodate a full size fillable anthropomorphic torso phantom and move it in three dimensions, whereas the Quasar respiratory motion phantom simply moves a cylindrical insert with a single source hot spot in only one dimension which is the superior/inferior direction. Quasar also produces a respiratory motion platform that can move a fillable anthropomorphic torso phantom in only two dimensions with the lateral motion limited to $1.0 \mathrm{~cm}$.

In this communication we have described a three dimensional drive system that could rigidly move a fillable phantom in a periodical elliptical path in three dimensions for use in SPECT and PET imaging. The frequency of motion could be varied manually in a calibrated and reproducible manner during the scan. It should be noted that the amplitude of the movement was set according to a review of the literature and could only be changed by modification of the cams on the 3D phantom drive system.

One useful aspect of the 3D drive system design is the ability to use it with many of the commercially available anthropomorphic phantoms. The device also neatly fits onto the patient beds of most diagnostic imaging equipment, such as gamma camera, SPECT, PET, CT and hybrid scanners.

From our preliminary analysis of the planar data, we can conclude that the 3D phantom drive system could be used to assess the effect of subject motion. The use of such a phantom should help quantitatively to assess the effectiveness of different motion correction techniques that are used for dynamic planar, SPECT and PET and has been used for 4D-CT imaging where it demonstrated sufficient accuracy of motion amplitude for high resolution studies. We intend to study the effect of respiratory motion on the determination of the target volumes in SPECT and PET images for 

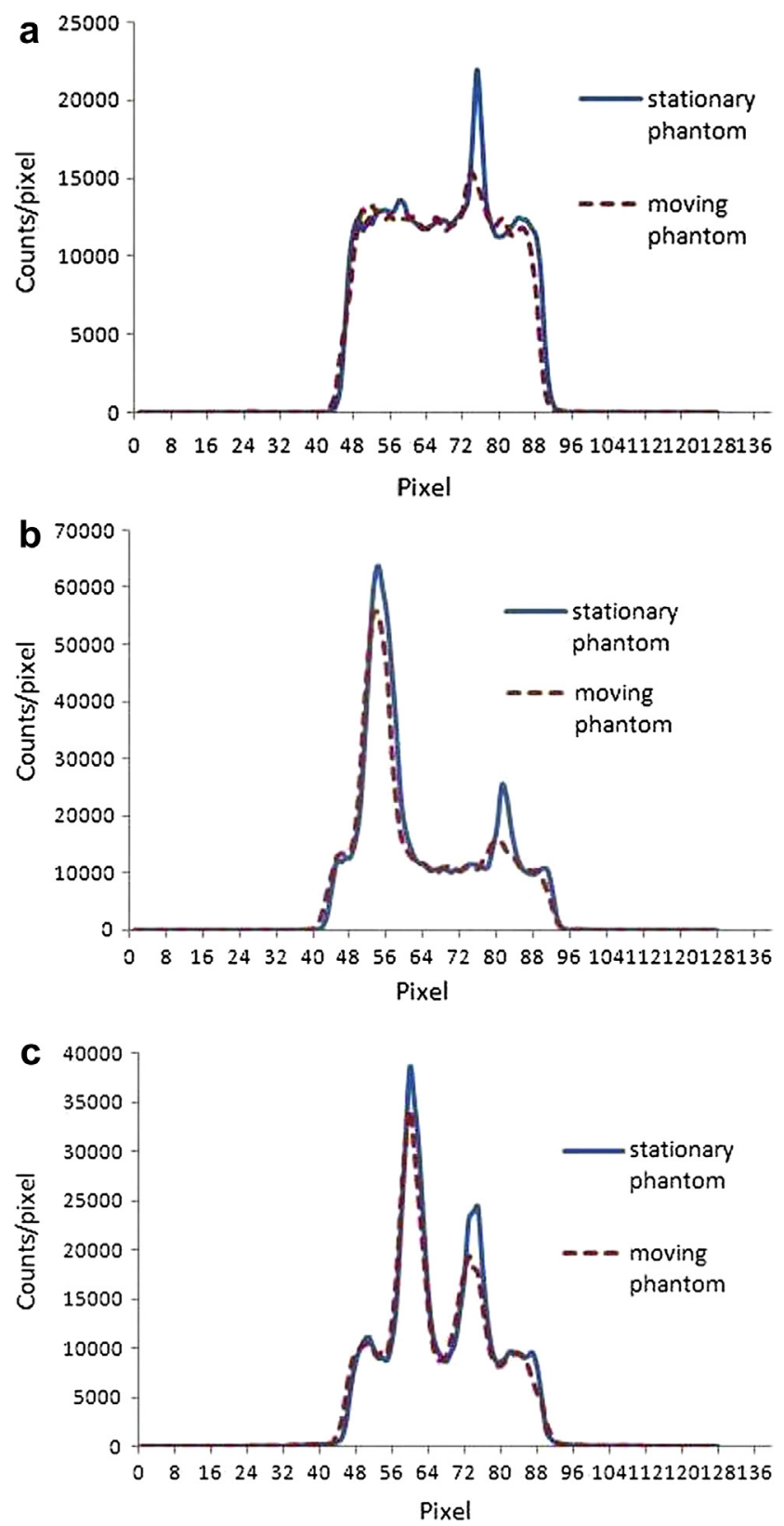

Figure 8. Horizontal profiles from transverse slices of the phantom showed reduced quality due to motion; Blue stationary image; dashed red moving image (a) the profiles through the smallest sphere $(15.9 \mathrm{~mm})$; (b) the profiles through the two middle spheres ( 38 \& $19.1 \mathrm{~mm}$ ); and (c) the profiles through the two bottom spheres in the phantom ( $31.8 \& 25.4 \mathrm{~mm}$ ). (For interpretation of the references to colour in this figure legend, the reader is referred to the web version of this article.)

Table 1

Review of previous studies.

\begin{tabular}{|c|c|c|c|}
\hline Study & $\begin{array}{l}\text { Motion } \\
\text { amplitude } \\
(\mathrm{mm})\end{array}$ & Direction of motion & $\begin{array}{l}\text { Time period } \\
\text { of one cycle (s) }\end{array}$ \\
\hline$[15]$ & $10,20 \& 30$ & Cranio-caudal (longitudinal) plane & NA \\
\hline [9] & 40 & $\begin{array}{l}\text { Movement at } 45^{\circ} \text { to the } \\
\text { Cranio-caudal direction }\end{array}$ & 3.74 \\
\hline [4] & 20 & $\begin{array}{l}\text { Cranio-caudal (longitudinal) } \\
\text { and anterior-posterior planes }\end{array}$ & 5.8 \\
\hline [16] & $10,20 \& 30$ & Cranio-caudal (longitudinal) plane & 4 \\
\hline [17] & $10 \& 20$ & Cranio-caudal (longitudinal) plane & NA \\
\hline [18] & Up to 38 & Cranio-caudal (longitudinal) plane & $1.75-6.61$ \\
\hline [14] & $5,10 \& 15$ & Three orthogonal planes & NA \\
\hline
\end{tabular}

radiotherapy treatment planning, and to evaluate the impact of respiratory motion on $\mathrm{CT}$ attenuation correction during SPECT or PET imaging.

For the purpose of acquiring gated data, the Anzai (Anzai AZ733V Respiratory Gating System; Anzai Medical Co.) and RPM (Varian Real-time Position Management; Varian Medical System) gating systems have been investigated and these are compatible with our system.

Although the prototype is fairly bulky, it is envisaged that future refinement will reduce the size and weight once further modifications have been made.

\section{Acknowledgements}

The authors wish to thank Elaine Blackshaw and Evelyn Shin for their help with the data acquisition.

\section{References}

[1] Giraud P, Yorke E, Ford EC, Wagman R, Mageras GS, Amols H, et al. Reduction of organ motion in lung tumors with respiratory gating. Lung Cancer 2006;51(1):41-51.

[2] Liu C, Pierce LA, Alessio AM, Kinahan PE. The impact of respiratory motion on tumor quantification and delineation in static PET/CT imaging. Physics in Medicine and Biology 2009;54(24):7345-62.

[3] Giraud P, De Rycke Y, Dubray B, Helfre S, Voican D, Guo L, et al. Conformal radiotherapy (CRT) planning for lung cancer: analysis of intrathoracic organ motion during extreme phases of breathing. International Journal Radiation Oncology Biology Physics 2001;51(4):1081-92.

[4] Vines DC, Keller H, Hoisak JDP, Breen SL. Quantitative PET comparing gated with nongated acquisitions using a NEMA phantom with respiratorysimulated motion. Journal of Nuclear Medicine Technology 2007;35(4): 246-51

[5] Martinez-Moller A, Souvatzoglou M, Navab N, Schwaiger M, Nekolla SG. Artifacts from misaligned CT in cardiac perfusion PET/CT studies: frequency, effects, and potential solutions. Journal of Nuclear Medicine 2007;48(2): 188-93.

[6] Roach PJ, Bailey DL, Harris BE. Enhancing lung scintigraphy with singlephoton emission computed tomography. Seminars in Nuclear Medicine 2008;38(6):441-9.

[7] Schäfers KP, Stegger L. Combined imaging of molecular function and morphology with PET/CT and SPECT/CT: image fusion and motion correction. Basic Research in Cardiology 2008;103(2):191-9.

[8] Nioutsikou E, Symonds-Tayler JRN, Bedford JL, Webb S. Quantifying the effect of respiratory motion on lung tumour dosimetry with the aid of a breathing phantom with deforming lungs. Physics in Medicine and Biology 2006;51(14): 3359.

[9] Mori S, Endo M, Kohno R, Minohara S. Improved motion compensation in 3D-CT using respiratory-correlated segment reconstruction: diagnostic and radiotherapy applications. British Journal of Radiology 2006;79(945): 745-55.

[10] Partridge M, Spinelli A, Ryder W, Hindorf C. The effect of [beta]+ energy on performance of a small animal PET camera. Nuclear Instruments and Methods in Physics Research Section A: Accelerators, Spectrometers, Detectors and Associated Equipment 2006;568(2):933-6.

[11] Bergmann $\mathrm{H}$. Test phantoms in nuclear medicine: planar gamma camera. Radiation Protection Dosimetry 1993;49(1-3):285-93.

[12] Meadows A, Hogg P. An analysis of motion correction for 99Tcm DMSA renal imaging in paediatrics. Radiography 2007;13(2):109-21.

[13] Ravindran P, Mahata A, Babu ES. Phantom for moving organ dosimetry with gel. Journal of Physics: Conference Series 2009;164(1):012057.

[14] Mutaf YD, Antolak JA, Brinkmann DH. The impact of temporal inaccuracies on 4DCT image quality. Medical Physics 2007;34(5):1615-22.

[15] Pitman AG, Kalff V, Van Every B, Risa B, Barnden LR, Kelly MJ. Effect of mechanically simulated diaphragmatic respiratory motion on myocardial SPECT processed with and without attenuation correction. Journal of Nuclear Medicine 2002:43(9):1259-67.

[16] Okubo M, Nishimura Y, Nakamatsu K, Okumura M, Shibata T, Kanamori S, et al. Static and moving phantom studies for radiation treatment planning in a positron emission tomography and computed tomography (PET/CT) system. Annals of Nuclear Medicine 2008;22(7):579-86.

[17] El Naqa I, Low DA, Bradley JD, Vicic M, Deasy JO. Deblurring of breathing motion artifacts in thoracic PET images by deconvolution methods. Medica Physics 2006;33(10):3587-600.

[18] Abdelnour AF, Nehmeh SA, Pan T, Humm JL, Vernon P, Schoder H, et al. Phase and amplitude binning for $4 \mathrm{D}-\mathrm{CT}$ imaging. Physics in Medicine and Biology 2007;52(12):3515-29. 